\title{
Prognosis of Early Stage Cervical Cancer According to Patterns of Recurrence
}

\author{
Yuanyuan Chen ${ }^{1,2}$ \\ Yiming $\mathrm{Zhu}^{3}$ \\ Jinchang $\mathrm{Wu}^{\prime}$ \\ 'Department of Radiation, The Affiliated \\ Suzhou Hospital of Nanjing Medical \\ University and Second Affiliated Hospital \\ of Xuzhou Medical University, Suzhou, \\ Jiangsu, People's Republic of China; \\ ${ }^{2}$ Department of Radiation, Women's \\ Hospital, School of Medicine, Zhejiang \\ University, Hangzhou, Zhejiang, People's \\ Republic of China; ${ }^{3}$ Department of \\ Gynecologic Oncology, Cancer Hospital \\ of University of Chinese Academy of \\ Sciences, Hangzhou, Zhejiang, People's \\ Republic of China
}

Correspondence: Jinchang Wu Department of Radiation, The Affiliated Suzhou Hospital of Nanjing Medical University and Second Affiliated Hospital of Xuzhou Medical University, Suzhou, Jiangsu, People's Republic of China Email wjinchang@sina.com
Purpose: Patterns of recurrence in cervical cancer may be useful as prognostic indicators. The aim of the present study was to determine the value of patterns of recurrence for predicting prognosis of early-stage cervical cancer.

Patients and Methods: Of the 1934 patients diagnosed with primary cervical cancer between August 2008 and July 2013, 167 experienced recurrence after radical hysterectomy, including pelvic lymphadenectomy, and adjuvant postoperative treatment. The patterns of recurrence were classified into four groups: central, pelvic, distant only, and combined metastases, and the relationship between patterns of recurrence and prognosis was evaluated.

Results: The patterns of lung only (21.6\%), central (21.0\%), and pelvic recurrence $(17.4 \%)$ were the most common sites, followed by distant lymph nodes and lung with other sites. The longest 5-year survival period occurred in patients with central recurrence $(70.5 \%)$, followed by distant lymph nodes $(58.4 \%)$, peritoneum $(58.3 \%)$, and lung only $(36.8 \%)$. Late recurrence was detected in 28 patients $(1.4 \%)$, who showed a better prognosis than those with early recurrence $(\mathrm{p}=0.003)$.

Conclusion: The patterns of recurrence help to predict prognosis. A central pattern of recurrence, distant lymph node recurrence, and peritoneal recurrence were associated with favorable outcomes after salvage therapy; however, patients who suffered other recurrent patterns, along with early recurrence, require more effective therapeutic strategies to improve survival.

Keywords: central, cervical cancer, pelvic, prognosis, recurrence

\section{Introduction}

Invasive cervical cancer (ICC) is the fourth most common malignancy and the fourth leading cause of cancer-related death in women worldwide. ${ }^{1}$ Radical hysterectomy plus pelvic node dissection (RHND), with or without para-aortic lymphadenectomy or radiotherapy are the standard treatments for early-stage disease. $^{2}$

However, 10-18\% of patients develop recurrent disease, which occurs within 3 years in most patients; $60 \%$ of cases show recurrence at a distant site with or without locoregional lesions, and these patients are generally considered

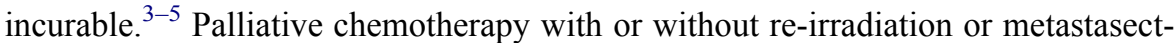
omy to reduce symptoms is used to improve prognosis. ${ }^{6,7}$ Postoperative assessment is essential for identifying the characteristics and patterns of recurrence and to optimize the therapeutic strategies. The aim of this retrospective study was to analyze the patterns of recurrence for predicting the prognosis of patients. 


\section{Patients and Methods}

All consecutive patients diagnosed with Federation International of Gynecology and Obstetrics (FIGO) stage I-IIA2 ICC treated at Cancer Hospital of University of Chinese Academy of Sciences, Zhejiang Province, China between August 2008 and July 2013 were analyzed. Clinical data were extracted from the institution's electronic database after obtaining informed consent from all patients. Follow-up data were obtained through outpatient department visits. The Medical Ethics Committee of Cancer Hospital of University of Chinese Academy of Sciences approved the study. This study was conducted in accordance with the Declaration of Helsinki.

All patients underwent primary surgery consisting of radical hysterectomy with pelvic lymphadenectomy and/or para-aortic lymphadenectomy. Patients with postoperative pathological risk factors, such as bulky tumor size, deep stromal invasion (DSI $\geq 1 / 2)$, lymphovascular space invasion (LVSI), lymphatic metastases, and parametrial and surgical margin involvement were advised to receive adjuvant radiotherapy (RT) or concurrent chemoradiotherapy (CCRT). RT consisted of 45-50 Gy of external beam radiotherapy with or without platinum-based concurrent chemotherapy.

The patterns of recurrence were classified into four groups: central (cervix or vaginal stump), pelvic (pelvic lymph nodes or pelvic side wall), distant only, and combined metastases. Distant only was defined as all recurrent lesions located outside the previous irradiation field. Combined metastases included recurrent focal lesions located within the previous irradiation field. Late recurrence was defined as relapse beyond 3 years.

Statistical analyses were performed using the SPSS 16.0 software package (IBM, Armonk, NY, USA). Summary statistics are presented as frequencies and percentages. Overall survival (OS) and relapse-free survival (RFS) were determined using the Kaplan-Meier method. $\mathrm{P}<0.05$ was considered statistically significant.

\section{Results}

\section{Patient Characteristics}

As of the data cutoff on Aug. 31, 2016, the median followup for overall survival was 52.2 months, and $45(2.3 \%)$ patients lost to follow-up. Of 1934 eligible patients, 534, 602, and 798 were treated with postoperative RT, CCRT, and observation, respectively. Recurrence developed in 167 individuals, including $81(13.46 \%)$ in the CCRT group, $32(5.99 \%)$ in the RT group, and $45(5.64 \%)$ in the observation group (Figure 1). The median age of the patients was 47 years (range, 28-73 years); 133 patients had squamous cell carcinoma (SCC) and 16 patients had adenocarcinoma (ADC). There were 82 patients with FIGO stage IB and 85 patients with stage II ICC. Among them, recurrence occurred within 3 years after RHND in $139(83.23 \%)$ patients and beyond 3 years after RHND in $28(16.77 \%)$ patients (Table 1).

\section{Recurrence Patterns}

Of the 167 total recurrences, distant only was the most common (52.7\%) site, followed by central (21.0\%), pelvic

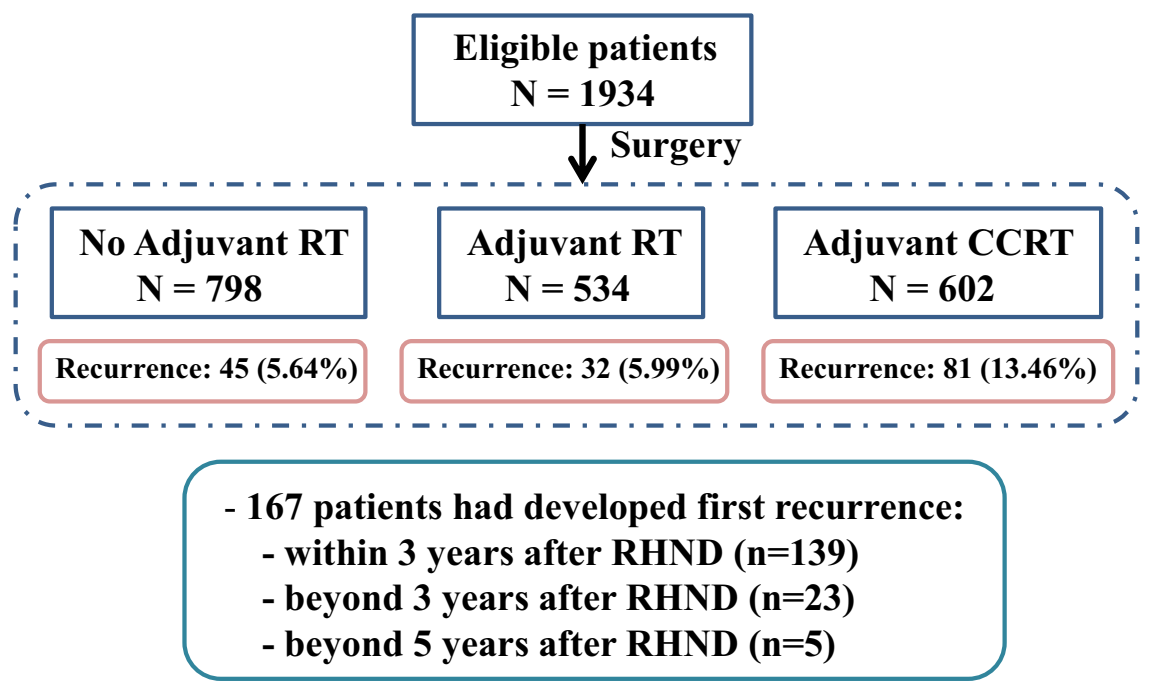

Figure I Flow diagram of patients cohort description.

Abbreviations: RT, radiotherapy; CCRT, concurrent chemoradiotherapy; RHND, radical hysterectomy plus pelvic node dissection. 
Table I Patient Characteristics and Clinical Outcomes of Patients with Early and Late Recurrence

\begin{tabular}{|c|c|c|c|}
\hline Variable & Total n (\%) & Early Recurrence $n$ & Late Recurrence n (\%) \\
\hline \multicolumn{4}{|l|}{ Age } \\
\hline$\leq 50 y$ & 101 & 87 & 14 \\
\hline$>50 y$ & 66 & 52 & 14 \\
\hline \multicolumn{4}{|l|}{ FIGO Stage } \\
\hline I & 82 & 69 & 13 \\
\hline$|\mathrm{B}|$ & 69 & 57 & $12(92.3 \%)$ \\
\hline IB2 & 13 & 12 & 1 \\
\hline ॥ & 85 & 70 & 15 \\
\hline$\| \mathrm{AI}$ & 61 & 46 & $15(100 \%)$ \\
\hline $\mathrm{IIA} 2$ & 24 & 24 & 0 \\
\hline \multicolumn{4}{|l|}{ Histology } \\
\hline SCC & 133 & 111 & 22 \\
\hline ADC & 16 & 13 & 3 \\
\hline Others & 18 & 15 & 3 \\
\hline \multicolumn{4}{|l|}{ Adjuvant therapy } \\
\hline No & 54 & 44 & 10 \\
\hline Radiation & 32 & 26 & 6 \\
\hline CCRT & 81 & 69 & 12 \\
\hline \multicolumn{4}{|l|}{ Pattern of recurrence } \\
\hline Central & $35(21)$ & 29 & 6 \\
\hline Pelvic & $30(17.4)$ & 26 & 4 \\
\hline Distant only & $87(52.7)$ & 71 & 16 \\
\hline Distant LN & 23 & & \\
\hline Liver \pm other sites & 6 & & \\
\hline Lung \pm other sites & 11 & & \\
\hline Bone \pm other sites & 2 & & \\
\hline Peritoneum \pm other sites & 9 & & \\
\hline Lung only & 36 & & \\
\hline Combined & $15(9)$ & 13 & 2 \\
\hline
\end{tabular}

Abbreviations: FIGO, Federation of International Gynecology and Obstetrics; SCC, squamous cell carcinoma; ADC, adenocarcinoma; ASC, adenosquamous; CCRT, concurrent chemoradiotherapy.

(17.4\%), and combined (9.0\%) subgroups. Distant only recurrence was divided into distant lymph nodes $(\mathrm{n}=$ 23 ), liver or lung with other sites ( $n=6$ and 11), bone or peritoneum with or without other sites, and lung only $(\mathrm{n}=$ 36). The most frequent pattern of recurrence was lung only (36 patients, $21.56 \%$ ), followed by central (35 patients, $20.96 \%$ ), pelvic (30 patients, 17.96\%), and other sites. Number of patients with positive nodes, parametrium infiltration, LVSI and related recurrent rate are shown in Supplementary Table 1.

The 5-year OS and RFS rates were $91.5 \% \pm 0.7 \%$ and $91.3 \% \pm 0.7 \%$, respectively. The 5 -year OS rates for recurrent patients with FIGO stage I and II were 63.4\% and $52.6 \%$, respectively $(\mathrm{P}=0.279)$. Of these, 28 patients eventually developed late recurrence. The 5-year OS rates in patients with early- and late recurrence were $39.8 \% \pm$ $4.4 \%$ and $59.1 \% \pm 10.1 \%$, respectively $(\mathrm{P}=0.003)$ (Figure 2). Five patients recurred beyond 5 years after RHND. Investigation of OS according to the patterns of recurrence showed that patients with central recurrence $(70.5 \%)$ had the longest survival, followed by distant lymph nodes $(58.4 \%)$, peritoneum $(58.3 \%)$, lung only (36.8\%), and others (Figure 3). Survival of the group subdivided in type of adjuvant treatment and localization of recurrence are shown in Supplementary Table 2.

\section{Discussion}

"Prevention is better than cure" wrote Hippocrates, and this is especially applicable to cervical cancer (CC), the incidence and mortality of which are expected to decline 


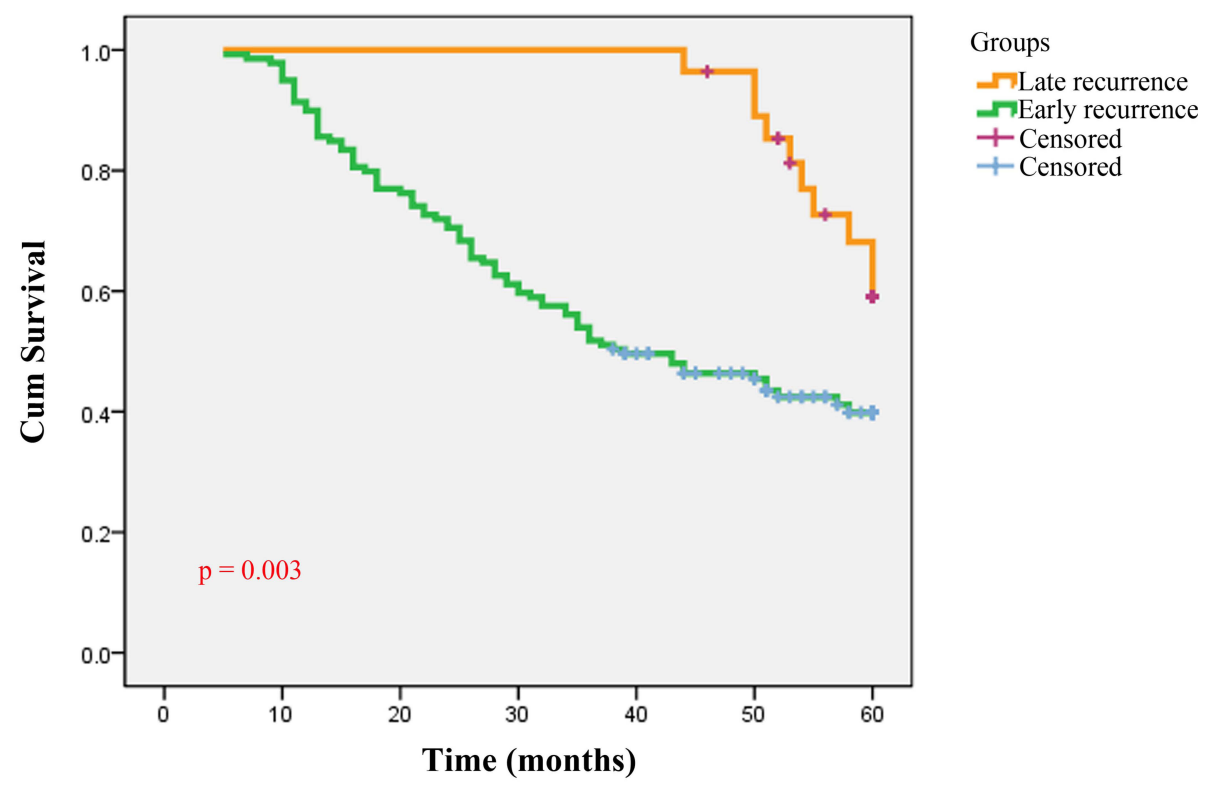

Figure 2 The 5 -year OS rates in patients with early- and late recurrence were $39.8 \% \pm 4.4 \%$ and $59.1 \% \pm 10.1 \%$, respectively $(P=0.003)$.

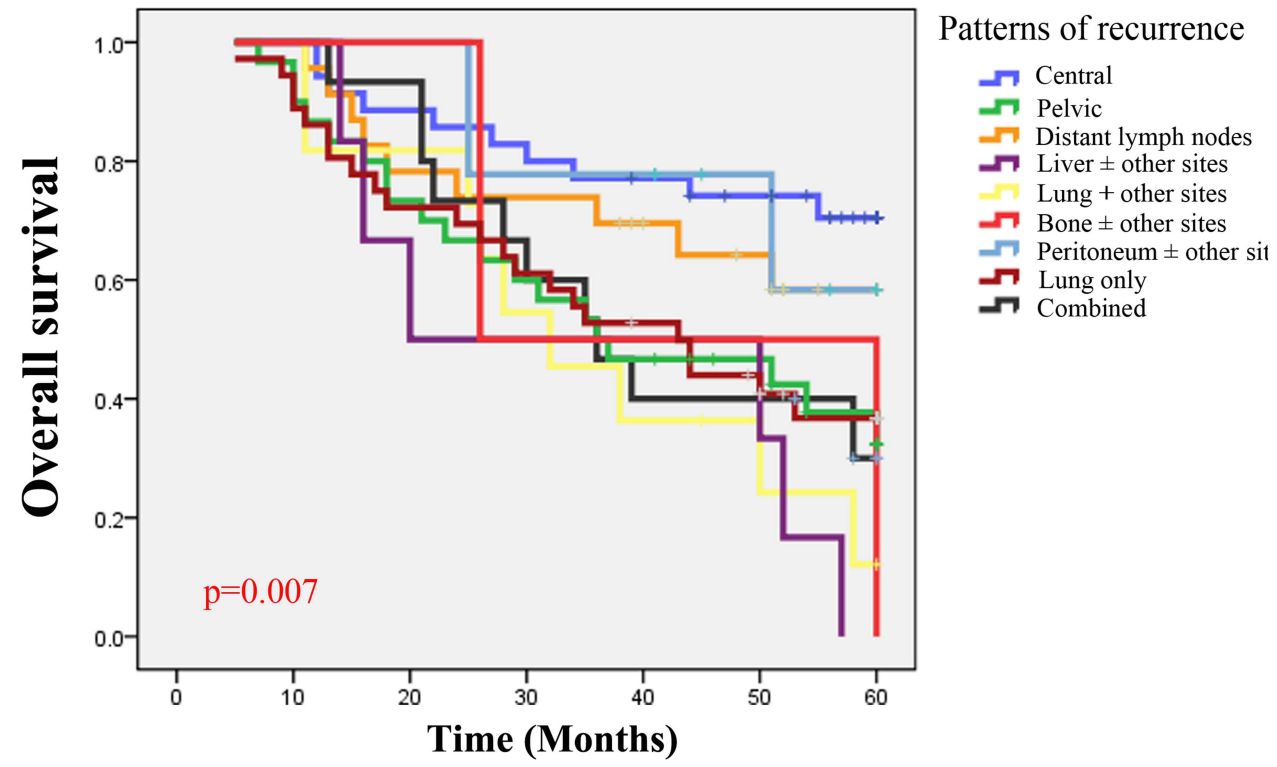

Figure 3 Overall survival according to pattern of recurrence. Investigation of OS according to the patterns of recurrence showed that patients with central recurrence (70.5\%) had the longest survival, followed by distant lymph nodes $(58.4 \%)$, peritoneum $(58.3 \%)$, lung only $(36.8 \%)$, and others $(p=0.007)$.

in forthcoming years due to population-based screening programs and Human Papillomavirus (HPV) vaccines. ${ }^{8}$ When detected early, $\mathrm{CC}$ can be cured by surgical excision and/or CCRT. However, de novo metastatic CC, as well as recurrent disease are not amenable to radical treatment, and are considered incurable with a dismal prognosis. ${ }^{9}$ Certain postoperative pathological factors are thought to influence risk of recurrence and survival, and are therefore recommended for adjuvant therapy, including RT or
CCRT. These high-risk factors include: lymph node metastasis, positive margin, and/or parametrial involvement, whereas intermediate risk factors are LVSI, DSI, and tumour size $>4 \mathrm{~cm}$. When one or more of these factors is detected, the 5ys survival may drop to between $50 \%$ and $70 \%{ }^{2}$

The treatment of recurrent $\mathrm{CC}$ remains largely ineffective and the prognosis is poor, underscoring the need to design more effective treatments. To design treatment strategies for 
recurrent $\mathrm{CC}$, many factors need to be considered, including the pattern of recurrence (central, pelvic, and/or distant), chemosensitivity, and performance status among others. The aim is to reduce the risk of future recurrence and minimize toxicity without sacrificing treatment efficacy. ${ }^{10}$ Pelvic exenteration is indicated in selected cases of central recurrence. ${ }^{10}$ Since we knew that nearly half of the patients with early-stage cervical cancer suffer from recurrence in the extra-pelvic regions, hence the main objective of CCRT is to reduce extra-pelvic recurrence. ${ }^{11}$ Although CCRT has shown benefits, tri-weekly cisplatin is not superior to a weekly regimen of adjuvant chemoradiation in patients with IB-IIB disease who underwent radical hysterectomy and pelvic lymphadenectomy. ${ }^{12-14}$ Smaniotto et al found that centrallocal recurrences have a better prognosis and OS rate than lateral-pelvic recurrences, which is possibly due to a better clinical response to chemoradiotherapy (45.4\% vs $18.2 \%) .{ }^{15}$ Targeted therapies have emerged as a promising treatment to improve the survival of patients with recurrent disease.

The rate of recurrence in patients with FIGO stage IB, IIA, IIB, III, and IVA has been estimated at 10\%, 17\%, $23 \%, 42 \%$, and $74 \%$, respectively. ${ }^{16}$ Approximately 30 $45 \%$ of recurrences after radical hysterectomy were of the central-pelvic-type, above the vaginal cuff and between the bladder and the rectum, and were associated with a better prognosis than pelvic wall recurrence. ${ }^{17}$ In the present study, the most common patterns of recurrence were lung only $(21.6 \%)$, central $(21.0 \%)$, and pelvic (17.4\%), followed by distant lymph nodes, lung with other sides, and others. Analysis of the survival outcomes of the recurrent subgroups showed that patients with a central recurrence pattern $(70.5 \%)$ had the longest survival, followed by distant lymph nodes $(58.4 \%)$, peritoneum $(58.3 \%)$, lung only $(36.8 \%)$, and others $(p=0.007)$. These findings suggested that patients with recurrence at distant lymph nodes, including supraclavicular lymph nodes, para-aortic lymph nodes, and inguinal lymph nodes, as well as those with peritoneal recurrence had a better prognosis and should receive salvage treatment. Moreover, cervical cancer receives the same standard treatments regardless pathologic types and recurrent patterns. A more aggressive therapeutic strategy different from current practice in cervical cancer is urgently required for low survival subgroups, to optimize clinical practice.

Recurrence of CC occurs mainly within 2-3 years from surgery; late recurrence is rare, and incidence rates vary from $0.8 \%$ to $4 \%$ in previous studies. ${ }^{18-20}$ In the present study, 167 patients $(8.63 \%)$ experienced recurrence, which occurred within 2 years in $71.26 \%$, within 3 years in $83.23 \%$, and beyond 5 years in $2.99 \%$ of patients. The percentage of recurrence beyond 5 years was similar to that reported previously. Late recurrence rate is related to the definition, as well as duration of follow-up. The 5-year OS rate was $59.1 \%$ in patients with late recurrence and $39.8 \%$ in patients with early recurrence $(\mathrm{P}=0.003)$, suggesting that $\mathrm{CC}$ with early recurrence was more aggressive than $\mathrm{CC}$ with late recurrence. Further efforts are needed to identify the risk factors for early or late recurrence, which may help design more effective treatment strategies for patients at high risk of early recurrence. ${ }^{21}$

The main strength of the present study was that all participants were under uniform treatment guidelines and quality control of pathology at the initial surgical treatment. However, the study had some limitations. First, this was a retrospective study and thus associated with potential selection bias. Second, variation in recurrence salvage therapy may have affected the survival rates.

In conclusion, the patterns of recurrence help to predict prognosis in early-stage CC. A central pattern of recurrence, distant lymph node recurrence, and peritoneal recurrence were associated with favorable outcomes after salvage therapy; however, patients who suffered other recurrent patterns, along with early recurrence, require more effective therapeutic strategies to improve survival.

\section{Ethics Statement}

This study was approved by The Medical Ethics Committee of Cancer Hospital of University of Chinese Academy of Sciences. All participants signed a term of informed consent prior to study commencement.

\section{Acknowledgment}

The authors are grateful to the MSD China Holding co., Ltd. Hangzhou, China for reviewing and proofreading. This work was funded by the Zhejiang Medical and Health Research Project (No. 2017KY535).

\section{Disclosure}

The authors report no conflicts of interest in this work.

\section{References}

1. Bray F, Ferlay J, Soerjomataram I, Siegel RL, Torre LA, Jemal A. Global cancer statistics 2018: GLOBOCAN estimates of incidence and mortality worldwide for 36 cancers in 185 countries. CA Cancer J Clin. 2018;68(6):394-424. doi:10.3322/caac.21492 
2. Abu-Rustum NR, Yashar CM, Bean S, et al. NCCN guidelines insights: cervical cancer, version 1.2020. J Natl Compr Canc Netw. 2020;18(6):660-666. doi:10.6004/jnccn.2020.0027

3. Nanthamongkolkul K, Hanprasertpong J. Longer waiting times for early stage cervical cancer patients undergoing radical hysterectomy are associated with diminished long-term overall survival. J Gynecol Oncol. 2015;26(4):262-269. doi:10.3802/jgo.2015.26.4.262

4. Fuglsang K, Petersen LK, Blaakaer J. Addressing challenges in future surveillance after surgery for early-stage cervical cancer. Int J Gynecol Cancer. 2015;25(2):309-314. doi:10.1097/IGC.0000000000000346

5. Hong JH, Tsai CS, Lai CH, et al. Recurrent squamous cell carcinoma of cervix after definitive radiotherapy. Int J Radiat Oncol Biol Phys. 2004;60(1):249-257. doi:10.1016/j.ijrobp.2004.02.044

6. Jeon W, Koh HK, Kim HJ, et al. Salvage radiotherapy for lymph node recurrence after radical surgery in cervical cancer. $J$ Gynecol Oncol. 2012;23(3):168-174. doi:10.3802/jgo.2012.23.3.168

7. Zolciak-Siwinska A, Bijok M, Jonska-Gmyrek J, et al. HDR brachytherapy for the reirradiation of cervical and vaginal cancer: analysis of efficacy and dosage delivered to organs at risk. Gynecol Oncol. 2014;132(1):93-97. doi:10.1016/j.ygyno.2013.10.018

8. Monk BJ, Tewari KS, Koh WJ. Multimodality therapy for locally advanced cervical carcinoma: state of the art and future directions. J Clin Oncol. 2007;25(20):2952-2965. doi:10.1200/JCO.2007.10.8324

9. Liontos M, Kyriazoglou A, Dimitriadis I, Dimopoulos MA, Bamias A. Systemic therapy in cervical cancer: 30 years in review. Crit Rev Oncol Hematol. 2019;137:9-17. doi:10.1016/j.critrevonc.2019.02.009

10. Peiretti M, Zapardiel I, Zanagnolo V, Landoni F, Morrow CP, Maggioni A. Management of recurrent cervical cancer: a review of the literature. Surg Oncol. 2012;21(2):e59-66. doi:10.1016/j.suronc.2011.12.008

11. Larson DM, Copeland LJ, Stringer CA, Gershenson DM, Malone JM Jr, Edwards CL. Recurrent cervical carcinoma after radical hysterectomy. Gynecol Oncol. 1988;30(3):381-387. doi:10.1016/ 0090-8258(88)90252-1

12. Rogers L, Siu SS, Luesley D, Bryant A, Dickinson HO. Radiotherapy and chemoradiation after surgery for early cervical cancer. Cochrane Database Syst Rev. 2012;5:CD007583.
13. Windschall A, Ott OJ, Sauer R, Strnad V. Radiation therapy and simultaneous chemotherapy for recurrent cervical carcinoma. Strahlenther Onkol. 2005;181(8):545e50. doi:10.1007/s00066-005-1340-8

14. Lee HN, Lee KH, Lee DW, Lee YS, Park EK, Park JS. Weekly cisplatin therapy compared with triweekly combination chemotherapy as concurrent adjuvant chemoradiation therapy after radical hysterectomy for cervical cancer. Int J Gynecol Cancer. 2011;21 (1):128-136. doi:10.1097/IGC.0b013e318200f7c5

15. Smaniotto D, D’Agostino G, Luzi S, et al. Concurrent 5-fluorouracil, mitomycin $\mathrm{C}$ and radiation with or without brachytherapy in recurrent cervical cancer: a scoring system to predict clinical response and outcome. Tumori. 2005;91:295e301.

16. Perez CA, Grigsby PW, Nene SM, et al. Effect of tumor size on the prognosis of carcinoma of the uterine cervix treated with irradiation alone. Cancer. 1992;69(11):2796e806. doi:10.1002/1097-0142(199 20601)69:11<2796::AID-CNCR2820691127>3.0.CO;2-O

17. Friedlander M, Grogan MUS. Preventative services task force. Guidelines for the treatment of recurrent and metastatic cervical cancer. Oncologist. 2002;7(4):342e7. doi:10.1634/theoncologist.20 02-0342

18. Goto T, Kino N, Shirai T, et al. Late recurrence of invasive cervical cancer: twenty years' experience in a single cancer institute. J Obstet Gynaecol Res. 2005;31(6):514-519. doi:10.1111/j.1447-0756.2005. 00343.x

19. Sobhan F, Sobhan F, Sobhan A. Recurrence of cancer cervix in patients treated by radical hysterectomy followed by adjuvant external beam radiotherapy. Bangladesh Med Res Counc Bull. 2010;36 (2):52-56. doi:10.3329/bmrcb.v36i2.6987

20. Hanprasertpong J, Jiamset I. Late recurrence of early stage cervical cancer more than 3 years after radical hysterectomy with pelvic node dissection. Oncol Res Treat. 2017;40(5):270-276. doi:10.1159/ 000464408

21. Kim TH, Kim MH, Kim BJ, Park SI, Ryu SY, Cho CK. Prognostic importance of the site of recurrence in patients with metastatic recurrent cervical cancer. Int J Radiat Oncol Biol Phys. 2017;98 (5):1124-1131. doi:10.1016/j.ijrobp.2017.03.029

\section{Publish your work in this journal}

Cancer Management and Research is an international, peer-reviewed open access journal focusing on cancer research and the optimal use of preventative and integrated treatment interventions to achieve improved outcomes, enhanced survival and quality of life for the cancer patient.
The manuscript management system is completely online and includes a very quick and fair peer-review system, which is all easy to use. Visit http://www.dovepress.com/testimonials.php to read real quotes from published authors. 